\title{
Atividade antimicrobiana e antibiofilme da Ilex paraguariensis: Uma revisão de
}

\section{literatura}

\author{
Ilex paraguariensis antimicrobial and antibiofilm activity: A literature review \\ Actividad antimicrobiana y antibiofilm de Ilex paraguariensis: Una revisión de la literatura
}

Recebido: 11/05/2021 | Revisado: 18/05/2021 | Aceito: 18/05/2021 | Publicado: 10/06/2021

\author{
Julia Wolfart Hindi \\ ORCID: https://orcid.org/0000-0003-2133-0925 \\ Universidade Federal de Santa Maria, Brasil \\ E-mail: hindiwjulia@gmail.com \\ Gabrielle da Silva Flores de Campos \\ ORCID: https://orcid.org/0000-0001-9106-4277 \\ Universidade Federal de Santa Maria, Brasil \\ E-mail: gabrielle.sfcampos@gmail.com \\ Laura Barzotto Klafki \\ ORCID: https://orcid.org/0000-0001-9487-358X \\ Universidade Federal de Santa Maria, Brasil \\ E-mail: laurinha.bk@gmail.com \\ Flávia Kolling Marquezan \\ ORCID: https://orcid.org/0000-0003-1505-2447 \\ Universidade Franciscana, Brasil \\ E-mail:flaviamarquezan@gmail.com \\ Natália Franco Brum \\ ORCID: https://orcid.org/0000-0001-6510-8889 \\ Universidade Federal de Santa Maria, Brasil \\ E-mail:natyfbrum@hotmail.com \\ Patricia Kolling Marquezan \\ ORCID: https://orcid.org/0000-0001-5061-6039 \\ Universidade Federal de Santa Maria, Brasil \\ E-mail: patimarquezan@hotmail.com
}

\begin{abstract}
Resumo
Nos últimos anos, o aumento da busca por alternativas medicinais e nutricionais no campo da saúde ocasionou a busca por plantas presentes no cotidiano da população. Consequentemente, diversas pesquisas almejavam avaliar o potencial e benefícios para a saúde. Portanto, o objetivo do estudo é realizar uma revisão de literatura sobre a potencial utilização medicinal e nutricional da planta Ilex paraguariensis. Foram realizadas pesquisas nas bases de dados PubMed, EMBASE, Biblioteca Virtual de Saúde (BVS), Cochrane Library, Web of Science, utilizando os descritores selecionados no MeSH, DeCs e Entree. As buscas foram realizadas no período de janeiro de 2020. O critério de inclusão utilizado englobava artigos dos últimos 20 anos encluindo revisões de literatura. Após as buscas foram selecionados 12 artigos para compor a revisão de literatura. A Ilex paraguariensis mostrou capacidade de atividade antimicrobiana e antiinflamatória. Os extratos da erva mate foram capazes de inibir os microrganismos: Salmonella enteritidis, Candida albicans, Proteus mirabilis, Pseudomas aeruginosa, Staphylococcus aureus, Staphylococcus epidermidis, Staphylococcus mutans, Listeria monocytogenes, Propionibacterium acnes, Enterobacteri cloacae, Bacillus cereus, Micrococcus flavus, Aspergillus fumigatus, Aspergillus versicolor, Aspergillus ochraceus, Aspergillus niger, Candida crusei, Penicillium funiculosum, Penicillium verrucosum var. ciclopium, Klebsiella pneumoniae, Enterobacter agglomerans, Enterobacter aerogeneses, Serratia marcesens, Proteus mirabilis. Porém, não houve consenso na literatura sobre atividade contra a Escherichia coli, Enterococcus faecalis e Acinetobacter baumannii. Assim, a erva mate apresenta um potencial antimicrobiano sob alguns microrganismos, mas ainda são necessários mais estudos para uma análise mais eficiente sobre a ação contra as bactérias e uma possível aplicação clínica.
\end{abstract}

Palavras-chave: Agentes anti-infecciosos; Ilex paraguariensis; Compostos fitoquímicos.

\begin{abstract}
In recent years, the increase in the search for medicinal and nutritional alternatives in the field of health has led to the search for plants present in the daily lives of the population. Consequently, several surveys aimed to assess the potential and health benefits. Therefore, the objective of the study is to carry out a literature review on the potential medicinal and nutritional use of the Ilex paraguariensis plant. Researches were carried out in the databases PubMed, EMBASE, Virtual Health Library (VHL), Cochrane Library, Web of Science, using the descriptors selected in MeSH, DeCs and Entree. The searches were carried out in the period of January 2020. The inclusion criterion used included
\end{abstract}


articles from the last 20 years, including literature reviews. After the searches, 12 articles were selected to compose the literature review. Ilex paraguariensis showed capacity for antimicrobial and anti-inflammatory activity. The extracts of yerba mate were able to inhibit microorganisms: Salmonella enteritidis, Candida albicans, Proteus mirabilis, Pseudomas aeruginosa, Staphylococcus aureus, Staphylococcus epidermidis, Staphylococcus mutans, Listeria monocytogenes, Propionibacterium acnes, Enterobacteri cloacae, Bacillus cereus, Micrococcus flavus, Aspergillus fumigatus, Aspergillus versicolor, Aspergillus ochraceus, Aspergillus niger, Candida crusei, Penicillium funiculosum, Penicillium verrucosum var. ciclopium, Klebsiella pneumoniae, Enterobacter agglomerans, Enterobacter aerogeneses, Serratia marcesens, Proteus mirabilis. However, there was no consensus in the literature on activity against Escherichia coli, Enterococcus faecalis and Acinetobacter baumannii. Thus, yerba mate has an antimicrobial potential under some microorganisms, but further studies are needed for a more efficient analysis of the action against bacteria and a possible clinical application.

Keywords: Anti-infective; Ilex paraguariensis; Phytochemicals.

\section{Resumen}

En los últimos años, el incremento en la búsqueda de alternativas medicinales y nutricionales en el campo de la salud ha llevado a la búsqueda de plantas presentes en la vida cotidiana de la población. En consecuencia, varios estudios tuvieron como objetivo evaluar el potencial y los beneficios para la salud. Por tanto, el objetivo del estudio es realizar una revisión de la literatura sobre el potencial uso medicinal y nutricional de la planta Ilex paraguariensis. Las investigaciones se realizaron en las bases de datos PubMed, EMBASE, Virtual Health Library (VHL), Cochrane Library, Web of Science, utilizando los descriptores seleccionados en MeSH, DeCs y Entree. Las búsquedas se realizaron en el período de enero de 2020. El criterio de inclusión utilizado incluyó artículos de los últimos 20 años, incluyendo revisiones de literatura. Después de las búsquedas, se seleccionaron 12 artículos para componer la revisión de la literatura. Ilex paraguariensis mostró capacidad de actividad antimicrobiana y antiinflamatoria. Los extractos de yerba mate fueron capaces de inhibir microorganismos: Salmonella enteritidis, Candida albicans, Proteus mirabilis, Pseudomas aeruginosa, Staphylococcus aureus, Staphylococcus epidermidis, Staphylococcus mutans, Listeria monocytogenes, Propionibacterium acnes, Enterobacteri cloacae, Bacillus cereus, Micrococcus flavus, Aspergillus fumigatus, Aspergillus versicolor, Aspergillus ochraceus, Aspergillus niger, Candida crusei, Penicillium funiculosum, Penicillium verrucosum var. ciclopium, Klebsiella pneumoniae, Enterobacter agglomerans, Enterobacter aerogeneses, Serratia marcesens, Proteus mirabilis. Sin embargo, no hubo consenso en la literatura sobre la actividad contra Escherichia coli, Enterococcus faecalis y Acinetobacter baumannii. Así, la yerba mate tiene un potencial antimicrobiano bajo algunos microorganismos, pero se necesitan más estudios para un análisis más eficiente de la acción contra las bacterias y una posible aplicación clínica.

Palabras clave: Agente anti-infecciosos; Ilex paraguariensis; Fitoquímicos.

\section{Introdução}

A erva-mate é uma planta da família Aquifoliaceae nativa da América do Sul, que cresce naturalmente em territórios paraguaios, uruguaios, argentinos e brasileiros e é cada vez mais utilizada nos Estados Unidos como ingrediente alimentar ou em suplementos dietéticos (Burris et al, 2011; Kungel et al, 2018). O pó da erva obtido da mistura de folhas secas e/ou caules finos da Ilex paraguariensis é usado para a preparação de várias bebidas estimulantes, chimarrão (extrato de água quente de folhas verdes secas; também chamado de mate nos países que falam espanhol), tererê (extrato de água fria de folhas verdes secas) e chá mate (extrato de água quente de folhas torradas) (Kungel et al, 2018).

A planta contém uma grande variedade de polifenóis, xantinas, derivados de cafeoil, saponinas e minerais (GonzalezGil et al, 2014) e as concentrações variam conforme as condições de crescimento e de processamento: a erva-mate cultivada em plantações apresentou com maiores concentrações de polifenóis do que aquelas que cresceram em florestas (Burris et al, 2011). Além disso, inúmeros efeitos farmacológicos benéficos na saúde humana já foram constatados, como a hipocolesterolemia, hepatoproteção, estimulação do sistema nervoso central, capacidade diurética, propriedades antimicrobianas e antioxidantes (Gonzalez-Gil et al, 2014). Estudos, ainda, sugerem que o consumo de folhas de erva-mate pode ter efeitos antiobesidade, antidiabéticos e quimiopreventivos, e, inclusive, pode ajudar na digestão (Burris et al, 2011).

Com o aumento da incidência de doenças transmitidas por alimentos contendo defensivos e a resistência a antibióticos há uma alta demanda por produtos mais naturais, bem como a necessidade de encontrar novos antimicrobianos capazes de combater, inclusive, essas bactérias multirresistentes a medicamentos (Davidson et al, 2011; Noureddine, 2018; Bezerra M et al., 2020; Brum N. et al., 2020; Bezerra M. et al., 2021). Antimicrobianos de origem vegetal podem ter inúmeras propriedades 
terapêuticas e potenciais antifúngicos, antiprotozoários, anti-helmínticos, antivirais e antibiofilme, tendo podencial de uso em odontologia (Francisco et al.2010, Filip et al, 2010). Relatórios recentes têm mostrado que um extrato aquoso de chá de ervamate, da planta Ilex paraguariensis, é bactericida e inibidor do crescimento de patógenos bacterianos como Enterobacter cloacae, Staphylococcus aureus, Streptococcus mutans, Listeria monocytogenes e Salmonella enteritidis (Burris, K. et al., 2012; Martin, J. et al., 2013) e pode conter um ou mais novos compostos antimicrobianos, como os metabólitos secundários derivados do ácido clorogênico (Hongpattarakere \& Johnson, 1999, Rempe et al, 2015).

Diante da importância do assunto, o objetivo desse estudo foi isolar, identificar e avaliar as atividades antimicrobianas de extratos da Ilex paraguariensis em cepas de bactérias Gram-positivas e Gram-negativas com diferentes perfis de suscetibilidade, incluindo organismos com altos níveis de resistência.

\section{Metodologia}

A revisão de literatura seguiu os preceitos do estudo descritivo de caráter qualitativo por meio de uma pesquisa bibliográfica na literatura científica. O método qualitativo por possibilitar uma melhor interpretação e inferência de opiniões sobre o assunto estudado foi utilizado pelos pesquisadores (Pereira et al., 2018). Buscou-se sintetizar o conhecimento sobre a atividade antimicrobiana e antibiofilme da espécie Ilex paraguariensis por meio de um levantamento bibliográfico em cinco bases de dados distintas: PubMed, Cochrane Library, EMBASE, Web of Science, BVS, sendo incluídos artigos dos últimos 20 anos e que abordassem propriedades antibiofilme e antimicrobiana da planta. Foram excluídas revisões de literatura e relatos de caso, além de estudos que não contemplassem o espaço temporal proposto nem a temática do presente trabalho.

Utilizou-se os descritores previamente selecionados a partir das plataformas MeSH, DeCS e Emtree, sendo adaptados para cada base de dados escolhida. As buscas foram realizadas por uma pesquisadora no período de Janeiro de 2021. Os termos buscados, bem como suas combinações por meio dos operadores booleanos “AND" e "OR" estão descritos na Quadro 1 juntamente com os resultados e o número de estudos selecionados.

Quadro 1: Combinação dos descritores nas bases de dados e resultados.

\begin{tabular}{|c|c|}
\hline $\begin{array}{l}\text { PubMed/MEDLINE: } \\
((((((((\text { erva mate) OR (Yerba MateS)) OR (Mates, Yerba)) OR (Yerba Mate)) OR (Mate, Yerba)) OR } \\
\text { (paraguarienses, Ilex)) OR (Ilex paraguarienses)) OR (ilex paraguariensis)) OR (paraguariensis, ilex) AND } \\
(((((((((((((((((((\text { Agents, Anti-Infective) OR (Anti Infective Agents)) OR (Antiinfective Agents)) OR (Anti- } \\
\text { Infective Agents)) OR (Agent, Antimicrobial)) OR (Antimicrobial Agent)) OR (Microbicide)) OR (Anti Microbial } \\
\text { Agents)) OR (Agents, Anti-Microbial)) OR (Agents, Antiinfective)) OR (Anti-Microbial Agents)) OR (Agents, } \\
\text { Antimicrobial)) OR (Antimicrobial Agents)) OR (Anti Microbial Agent)) OR (Agent, Anti-Microbial)) OR (Anti- } \\
\text { Microbial Agent)) OR (Microbicides)) OR (Anti Infective Agent)) OR (Agent, Anti-Infective)) OR (Anti-Infective } \\
\text { Agent)) OR (antibiofilm) }\end{array}$ & \\
\hline $\begin{array}{l}\text { EMBASE } \\
\text { (('antiinfective agent'/exp OR 'anti bacterial agent' OR 'anti bacterial agents' OR 'anti infective agents' OR 'anti } \\
\text { infectives, otic' OR 'anti-bacterial agents' OR 'anti-infective agents' OR 'anti-infectives, otic' OR 'antibacterial' OR } \\
\text { 'antibacterial agent' OR 'antibacterial drug' OR 'antibacterial soap' OR 'antibacterial spectrum' OR 'antiinfective } \\
\text { agent' OR 'antiinfectives, otic' OR 'antimicrobial' OR 'antimicrobial agent' OR 'antimicrobial compound' OR } \\
\text { 'antimicrobial drug' OR 'antimicrobial factor' OR 'antiseptic' OR 'antiseptic agent' OR 'antiseptic cream' OR } \\
\text { 'antiseptic foam' OR 'antiseptic soap' OR 'chemotherapeutic agent' OR 'chemotherapeutic drug' OR } \\
\text { 'chemotherapeutica' OR 'microbiological agent') AND ('ilex paraguariensis'/exp OR 'ilex paraguariensis' OR 'yerba } \\
\text { mate') }\end{array}$ & $\begin{array}{c}\# 1 \text { AND \#2 } \\
203\end{array}$ \\
\hline $\begin{array}{l}\text { COCHRANE } \\
(((((((((\text { erva mate) OR (Yerba MateS)) OR (Mates, Yerba)) OR (Yerba Mate)) OR (Mate, Yerba)) OR } \\
(\text { paraguarienses, Ilex)) OR (Ilex paraguarienses)) OR (ilex paraguariensis)) OR (paraguariensis, ilex) AND } \\
(((((((((((((((((((\text { Agents, Anti-Infective) OR (Anti Infective Agents)) OR (Antiinfective Agents)) OR (Anti- } \\
\text { Infective Agents)) OR (Agent, Antimicrobial)) OR (Antimicrobial Agent)) OR (Microbicide)) OR (Anti Microbial } \\
\text { Agents)) OR (Agents, Anti-Microbial)) OR (Agents, Antiinfective)) OR (Anti-Microbial Agents)) OR (Agents, }\end{array}$ & $\begin{array}{c}\text { AND \#2 } \\
0\end{array}$ \\
\hline
\end{tabular}




\begin{tabular}{|c|c|}
\hline $\begin{array}{l}\text { Antimicrobial)) OR (Antimicrobial Agents)) OR (Anti Microbial Agent)) OR (Agent, Anti-Microbial)) OR (Anti- } \\
\text { Microbial Agent)) OR (Microbicides)) OR (Anti Infective Agent)) OR (Agent, Anti-Infective)) OR (Anti-Infective } \\
\text { Agent)) OR (antibiofilm) }\end{array}$ & \\
\hline $\begin{array}{l}\text { (Anti-Infecciosos OR Agentes Anti-Infecciosos OR Agentes Antimicrobianos OR Antimicrobianos Ação } \\
\text { Antimicrobiana OR Fármacos Anti-Infecciosos OR Microbicidas OR Anti-Infective Agents OR Anti-Microbial } \\
\text { Agents OR Antiinfective Agents OR Antimicrobial Agents OR Microbicides Antiinfecciosos OR Agentes } \\
\text { Antiinfecciosos OR Agentes Antimicrobianos OR Antimicrobianos OR Fármacos Antiinfecciosos OR } \\
\text { Microbicidas) AND (Ilex paraguariensis OR Erva-Mate OR Mate OR Ilex paraguariensis OR Mate, Yerba OR } \\
\text { Yerba Mate OR Ilex paraguariensis OR Yerba Mate) }\end{array}$ & $\begin{array}{c}\# 1 \text { AND \#2 } \\
241\end{array}$ \\
\hline $\begin{array}{l}\text { WEB OF SCIENCE } \\
\text { (Anti-Infecciosos OR Agentes Anti-Infecciosos OR Agentes Antimicrobianos OR Antimicrobianos Ação } \\
\text { Antimicrobiana OR Fármacos Anti-Infecciosos OR Microbicidas OR Anti-Infective Agents OR Anti-Microbial } \\
\text { Agents OR Antiinfective Agents OR Antimicrobial Agents OR Microbicides Antiinfecciosos OR Agentes } \\
\text { Antiinfecciosos OR Agentes Antimicrobianos OR Antimicrobianos OR Fármacos Antiinfecciosos OR } \\
\text { Microbicidas) AND (Ilex paraguariensis OR Erva-Mate OR Mate OR Ilex paraguariensis OR Mate, Yerba OR } \\
\text { Yerba Mate OR Ilex paraguariensis OR Yerba Mate) }\end{array}$ & $\begin{array}{c}1 \text { AND \#2 } \\
2\end{array}$ \\
\hline
\end{tabular}

Fonte: Autores (2021).

\section{Resultados e Discussão}

Após a vasta busca pela literatura, os artigos foram selecionados por título e resumo, eliminando as duplicatas posteriormente e realizando a leitura crítica dos estudos considerados elegíveis. Foram excluídos artigos pelos critérios de exclusão (fuga do tema, duplicatas ou não disponíveis on-line) até chegar aos artigos incluídos na revisão de literatura. Do total de estudos encontrados foram selecionados 12 artigos científicos que contemplavam a temática. A seleção seguindo critérios foi fundamental para a melhor confiabilidade e poder de generalização das conclusões desse estudo. Além disso, a descrição dos estudos selecionados foi sintetizada na forma de quadro (Quadro 1) para melhor visualização e conhecimento dos artigos.

Quadro 1: Artigos selecionados.

\begin{tabular}{|c|c|c|c|c|}
\hline AUTOR/ANO & TÍTULO & $\begin{array}{l}\text { PLANTA } \\
\text { UTILIZADA }\end{array}$ & $\begin{array}{l}\text { MICRORGANISMO } \\
\text { TESTADO }\end{array}$ & RESULTADOS \\
\hline $\begin{array}{l}\text { Girolometto G. et al., } \\
2009\end{array}$ & $\begin{array}{c}\text { Atividade antibacteriana } \\
\text { de extratos de erva-mate } \\
\text { (Ilex paraguariensis } \\
\text { A.St.-Hil.) }\end{array}$ & $\begin{array}{c}\text { Ilex } \\
\text { paraguariensis }\end{array}$ & $\begin{array}{l}\text { Staphylococcus aureus, } \\
\text { Enterococcus faecalis, } \\
\text { Salmonella enteritidis e } \\
\text { Escherichia coli. }\end{array}$ & $\begin{array}{l}\text { Ilex paraguariensis apresentou } \\
\text { capacidade de inativação sobre as } \\
\text { bactérias avaliadas, os extratos } \\
\text { originados através de destilação } \\
\text { etanólica mostraram resultados } \\
\text { ainda mais favoráveis. A } S \text {. } \\
\text { enteritidis foi o agente bacteriano } \\
\text { mais sensível, seguido de } E \text {. } \\
\text { faecalis. E com menores } \\
\text { sensibilidades } S \text {. aureus e } E \text {. coli. }\end{array}$ \\
\hline $\begin{array}{c}\text { De Biasi B. et al., } \\
2009\end{array}$ & $\begin{array}{l}\text { Atividade antimicrobiana } \\
\text { de extratos de folhas e } \\
\text { ramos de Ilex } \\
\text { paraguariensis (erva- } \\
\text { mate) }\end{array}$ & $\begin{array}{c}\text { Ilex } \\
\text { paraguariensis }\end{array}$ & $\begin{array}{c}\text { Candida albicans, } \\
\text { Escherichia coli, Proteus } \\
\text { mirabilis, Pseudomonas } \\
\text { aeruginosa, } \\
\text { Staphylococcus aureus e } \\
\text { Staphylococcus } \\
\text { epidermidis. }\end{array}$ & $\begin{array}{c}\text { C.albicans, P.mirabilis, } \\
\text { P.aeruginosa, } \\
\text { S. aureus e S.epidermidis } \\
\text { apresentaram sensibilidade aos } \\
\text { extratos, porém com variação aos } \\
\text { extratos expostos no sol. Não } \\
\text { houve atividade antimicrobiana } \\
\text { contra } E \text {. coli. }\end{array}$ \\
\hline
\end{tabular}




\begin{tabular}{|c|c|c|c|c|}
\hline $\begin{array}{c}\text { Battagim, J. et al., } \\
2011\end{array}$ & $\begin{array}{l}\text { Estudo comparativo do } \\
\text { efeito dos extratos verde e } \\
\text { água torrada de mate (Ilex } \\
\text { paraguariensis) na } \\
\text { atividade da } \\
\text { glicosiltransferase de } \\
\text { Streptococcus mutans. }\end{array}$ & $\begin{array}{c}\text { Ilex } \\
\text { paraguariensis }\end{array}$ & Streptococcus mutans. & $\begin{array}{l}\text { Foram notadas atividades } \\
\text { inibitórias em diferentes } \\
\text { concentrações de extratos da } \\
\text { folha verde e da folha torrada da } \\
\text { erva contra o } S \text {. mutans; os } \\
\text { extratos da folha torrada foram } \\
\text { inibidores mais efetivos do que } \\
\text { os da folha verde. }\end{array}$ \\
\hline Burris, et al., 2011 & $\begin{array}{c}\text { Atividade antimicrobiana } \\
\text { de extratos aquosos de } \\
\text { Erva Mate (Ilex } \\
\text { paraguariensis) contra } \\
\text { Escherichia coli O157: } \\
\text { H7 e Staphylococcus } \\
\text { aureus. }\end{array}$ & $\begin{array}{c}\text { Ilex } \\
\text { paraguariensis }\end{array}$ & $\begin{array}{c}\text { Escherichia coli } \mathrm{e} \\
\text { Staphylococcus aureus. }\end{array}$ & $\begin{array}{c}\text { Extratos aquosos dialisados de } \\
\text { erva-mate, mesmo em } \\
\text { concentrações relativamente } \\
\text { baixas, fornecem atividade } \\
\text { bactericida contra a } S \text {. aureus e a } \\
\text { E. coli. }\end{array}$ \\
\hline $\begin{array}{l}\text { Carelli G., et al., } \\
2011\end{array}$ & $\begin{array}{l}\text { Avaliação preliminar da } \\
\text { atividade antimicrobiana } \\
\text { do extrato de erva-mate } \\
\text { (Ilex paraguariensis A. } \\
\text { St.-Hil.) obtido pela } \\
\text { utilização de } \\
\mathrm{CO}_{2} \text { supercrítico }\end{array}$ & $\begin{array}{c}\text { Ilex } \\
\text { paraguariensis }\end{array}$ & $\begin{array}{c}\text { Acinetobacter baumannii, } \\
\text { Escherichia coli, } \\
\text { Pseudomonas aerugiosa, } \\
\text { Staphylococcus aureus } \mathrm{e} \\
\text { Enterococcus faecalis. }\end{array}$ & $\begin{array}{l}\text { Houve atividade antimicrobiana } \\
\text { in vitro contra as cepas de } S . \\
\text { aureus e } P \text {. aeruginosa, não } \\
\text { ocorrendo o mesmo para as cepas } \\
\text { de E. coli, E. faecalis e } \\
\text { A.baumannii. }\end{array}$ \\
\hline Burris, K. et al., 2012 & $\begin{array}{l}\text { Extratos aquosos de erva- } \\
\text { mate (Ilex } \\
\text { paraguariensis) como } \\
\text { antimicrobiano natural } \\
\text { contra Escherichia coli } \\
\text { O157: H7 em meio } \\
\text { microbiológico e suco de } \\
\text { maçã com pH } 6,0 .\end{array}$ & $\begin{array}{c}\text { Ilex } \\
\text { paraguariensis }\end{array}$ & Escherichia coli. & $\begin{array}{l}\text { Extratos aquosos de erva-mate } \\
\text { dialisados e liofilizados } \\
\text { demonstraram atividade } \\
\text { bactericida contra } E \text {. coli em um } \\
\text { meio microbiológico e baixas } \\
\text { concentrações de extratos } \\
\text { aquosos da erva fornecem } \\
\text { atividade antimicrobiana contra } \\
\text { E. coli no suco de maçã } \\
\text { modificado utilizado na pesquisa. }\end{array}$ \\
\hline Martin, J. et al., 2013 & $\begin{array}{l}\text { Atividade antimicrobiana } \\
\text { da erva-mate (Ilex } \\
\text { paraguariensis St. Hil.) } \\
\text { Contra patógenos } \\
\text { alimentares. }\end{array}$ & $\begin{array}{c}\text { Ilex } \\
\text { paraguariensis }\end{array}$ & $\begin{array}{c}\text { Staphylococcus aureus, } \\
\text { Listeria } \\
\text { monocytogenes, Salmonella } \\
\text { Enteritidis e Escherichia } \\
\text { coli }\end{array}$ & $\begin{array}{c}\text { Os extratos metanólico e } \\
\text { etanólico da erva-mate inibiram } \\
\text { todos os microrganismos } \\
\text { avaliados, exceto } E \text {. Coli; o } \\
\text { extrato inibiu o crescimento de } S . \\
\quad \text { Aureus e de } L . \\
\text { monocytogenes em pH } 7 \text { e } 8 .\end{array}$ \\
\hline $\begin{array}{c}\text { Gonzalez-Gil, } \\
\text { Francisco, et al., } 2013\end{array}$ & $\begin{array}{l}\text { A erva-mate aumenta o } \\
\text { crescimento de bactérias } \\
\text { probióticas in vitro, mas } \\
\text { como aditivo alimentar } \\
\text { não reduz a colonização } \\
\text { de Enteritidis in vivo. }\end{array}$ & $\begin{array}{c}\text { Ilex } \\
\text { paraguariensis }\end{array}$ & $\begin{array}{c}\text { Salmonella Enteritidis, } \\
\text { Salmonella Typhimurium, } \\
\text { Salmonella Senftenburg e } \\
\text { Bactérias Lácteas } \\
\text { (LAB):Lactobaciullus } \\
\text { acidophilus e Pediococcus. }\end{array}$ & $\begin{array}{l}\text { In vitro, a erva-mate possui } \\
\text { atividade antimicrobiana e } \\
\text { melhora o crescimento de } \\
\text { bactérias lácteas, porém, in vivo, } \\
\text { a erva-mate não diminuiu a } \\
\text { colonização da Salmonella } \\
\text { Enteriidis. }\end{array}$ \\
\hline $\begin{array}{l}\text { Tsai T.-H., et al., } \\
2015\end{array}$ & $\begin{array}{l}\text { Efeitos antimicrobianos e } \\
\text { antiinflamatórios in vitro } \\
\quad \text { de ervas contra } \\
\text { Propionibacterium acnes }\end{array}$ & $\begin{array}{c}\text { Ilex } \\
\text { paraguariensis }\end{array}$ & Propionibacterium acnes. & $\begin{array}{l}\text { A erva mate demostrou atividade } \\
\text { antibacteriana contra a bactéria } \\
\text { Propionibacterium acnes. }\end{array}$ \\
\hline $\begin{array}{l}\text { Rempe, C. et al., } \\
2015\end{array}$ & $\begin{array}{l}\text { Classificação } \\
\text { computacional de } \\
\text { pequenas moléculas de } \\
\text { erva-mate com base em } \\
\text { sua contribuição prevista } \\
\text { para a atividade } \\
\text { antibacteriana contra } \\
\text { Staphylococcus aureus } \\
\text { resistente à meticilina. }\end{array}$ & $\begin{array}{c}\text { Ilex } \\
\text { paraguariensis }\end{array}$ & Staphylococcus aureus. & $\begin{array}{l}\text { Os ácidos cítrico, cafeico e } \\
\text { clorogênico, o kaempferol e a } \\
\text { quercetina são compostos que } \\
\text { podem contribuir para a atividade } \\
\text { antimicrobiana do extrato de } \\
\text { erva-mate contra o S.aureus. }\end{array}$ \\
\hline
\end{tabular}




\begin{tabular}{|c|c|c|c|c|}
\hline $\begin{array}{l}\text { Kungel, P. et al., } \\
2018\end{array}$ & $\begin{array}{l}\text { Atividades antioxidante e } \\
\text { antimicrobiana de um } \\
\text { polissacarídeo purificado } \\
\text { da erva-mate (Ilex } \\
\text { paraguariensis). }\end{array}$ & $\begin{array}{c}\text { Ilex } \\
\text { paraguariensis }\end{array}$ & $\begin{array}{c}\text { Bactérias Gram-negativas } \\
\text { (Escherichia coli, } \\
\text { Enterobacter cloacae, } \\
\text { Salmonella enteritidis e } \\
\text { Salmonella typhimurium), } \\
\text { bactérias Gram-positivas } \\
\text { (Bacillus cereus, } \\
\text { Micrococcus flavus, } \\
\text { Staphylococcus aureus e } \\
\text { Listeria monocytogenes), e } \\
\text { microfungos Aspergillus } \\
\text { fumigatus, Aspergillus } \\
\text { versicolor, Aspergillus } \\
\text { ochraceus, Aspergillus } \\
\text { niger, Candida crusei, } \\
\text { Penicillium funiculosum e } \\
\text { Penicillium verrucosum } \\
\text { var. ciclopium. }\end{array}$ & $\begin{array}{l}\text { Teve efeitos antimicrobianos } \\
\text { contra } \text { E. cloacae, } \text { S. enteritidis, } \\
\text { S.typhimurium, B. cereus, } \\
\text { M.flavus, S.aureus, } \\
\text { L.monocytogenes, A.fumigatus, } \\
\text { versicolor, ochraceus, niger, } C \text {. } \\
\text { crusei, } \text { P.funiculosum e } P . \\
\text { verrucosum var. ciclopium; foi } \\
\text { considerado um forte inibidor } \\
\text { contra } B . \text { cereus, } M \text {. flavus, } E \\
\text { cloacae, } S . \text { enteritidis e } S . \\
\text { typhimurium e um inibidor fraco } \\
\text { de } E \text {. coli.; parece ser um forte } \\
\text { inibidor contra todos os fungos } \\
\text { testados, exceto para } A . \text { niger. }\end{array}$ \\
\hline $\begin{array}{l}\text { Noureddine T. , et al., } \\
2018\end{array}$ & $\begin{array}{l}\text { Atividade antibacteriana } \\
\text { de Ilex paraguariensis } \\
\text { (Yerba Mate) contra } \\
\text { bactérias Gram-positivas } \\
\text { e Gram-negativas }\end{array}$ & $\begin{array}{c}\text { Ilex } \\
\text { paraguariensis }\end{array}$ & $\begin{array}{l}\text { Acinetobacter baumannii, } \\
\text { Pseudomonas aeruginosa, } \\
\text { Staphylococcus aureus, } \\
\text { Escherichia coli, Klebsiella } \\
\text { pneumoniae, Enterococcus } \\
\text { faecalis, Enterobacter } \\
\text { agglomerans, Enterobacter } \\
\text { aerogenes, Serratia } \\
\text { marcesens, Escherichia coli } \\
\text { e Proteus mirabilis. }\end{array}$ & $\begin{array}{c}\text { A erva mate possui atividade } \\
\text { antibacteriana contra todas as } \\
\text { cepas testadas, entretanto, essa } \\
\text { atividade varia entre as bactérias } \\
\text { Gram-positivas e Gram- } \\
\text { negativas, sendo essa atividade } \\
\text { maior em bactérias Gram- } \\
\text { positivas. }\end{array}$ \\
\hline
\end{tabular}

Fonte: Autores (2021).

Nos estudos realizados pelo Girolometto et al. (2009), por meio de testes de diluição, com uma sistemática de tubos múltiplos, a planta Ilex paraguariensis mostrou capacidade antimicrobiana em alguns microrganismos, apresentando uma inativação significativa nessas bactérias, como: Staphylococcus aureus, Enterococcus faecalis, Salmonella enteritidis e Escherichia coli. Dentre essas, a Salmonella enteritidis foi a que mais demostrou sensibilidade ao extrato etanólico da ervamate. A ação contra a bactéria é importante porque a $S$. enteritis trata-se de um patógeno que causa infecções alimentares, sendo danosa ao ser humano. Assim Ilex paraguariensis mostra-se em potencial para um possível controle de Salmonelose, sendo apontada como o principal microrganismo envolvido nos surtos de doenças alimentares em geral, identificada em 7,5\% dos casos entre os anos de 2007 e 2016, no Brasil (Brasil 2016, Baptista et al., 2018).

Segundo De Biasi et al. (2009), a erva-mate é capaz de inativar algumas outras bactérias, como a Candida albicans, Proteus mirabilis, Pseudomonas aeruginosa, Staphylococcus aureus e Staphylococcus epidermidis. O estudo foi feito utilizando a metodologia de difusão do disco, com extratos hidro alcoólicos das folhas da erva- mate coletadas em dois ambientes: sem e com exposição da luz solar. Porém, não houve atividade contra bactéria Gram-negativa Escherichia coli, mesmo testando extratos de folhas e ramos da I. paraguariensis expostas ao sol. A E. coli é encontrada no trato gastrointestinal dos seres humanos, e pode causar intoxicações alimentares. Logo, a erva-mate não possui efeito antimicrobiano sobre essa bactéria e não possui capacidade de atenuar problemas relacionados a tal patógeno.

Nos estudos de Battagim,. et al. (2011) foram realizados testes para verificar as diferentes concentrações de extratos da folha verde e da folha verde torrada da I. paraguariensis em contato com S. mutans. Essa bactéria está diretamente ligada ao desenvolvimento do biofilme dentário patogênico e de doenças orais como a cárie e doença periodontal. O resultado dos testes foi que extratos da folha torrada inibem mais efetivamente do que os da folha verde sem torrar a enzima glicosiltransferase presente na bactéria e responsável pela formação de glucanos extracelulares insolúveis que possibilitam a adesão e coadesão microbiana. Além disso, vale ressaltar que está comprovado que os extratos destilados de N-hexano da erva mate são agentes antimicrobianos eficazes contra a bactéria oral $S$. mutans (Kubo et al., 1993). Assim, a relação da erva-mate com o $S$. mutans 
torna-se de interesse do cirurgião dentista, já que esse potencial pode ser utilizado pela área odontológica a fim de minimizar a presença da bactéria acidogênicas e acidúricas relacionadas ao biofilme dito cariogênico (Fejerskov et al., 2011).

Segundo os estudos dirigidos por Kellie P. Burris et al. (2011), a atividade antimicrobiana da erva mate contra os patógenos E. coli e S. aureus, que causam infecções e intoxicações alimentares, respectivamente, foi confirmada. Extratos aquosos dialisados de erva-mate foram utilizados com o objetivo de avaliar a inibição de crescimento e a atividade de inibição contra esses patógenos, concluindo que a atividade antimicrobiana da I. paraguariensis se deve aos compostos encontrados naturalmente nas folhas e caules da planta, como os polifenóis. Essa atividade contra a E. coli e a S. aureus foi obtida usando quantidades de chás inferiores ou comparáveis as quantidades de consumo típico da erva-mate (50g). Tal estudo é importante uma vez que reforça a ideia de que extrato aquoso da planta I. paraguariensis pode ser utilizada no tratamento de doenças bacterianas como gastroenterites e infecções urinarias, em razão do seu potencial antimicrobiano (Silva et al., 2012).

Salienta-se que os polifenóis encontrados na erva-mate são o ácido cafeico, cafeína, derivados de cafeoyl, ácido clorogênico, ácido caffeoylshikimic, ácido feruloylquiniico, kaempferol, quercetina, ácido quínico rutina e teobromina (Heck et al., 2007; Marques et al., 2009), os quais podem contribuir para a atividade antimicrobiana contra a E. coli e a S. aureus, sendo que os ácidos cafeico e clorogênico, nas suas formas puras, demonstraram atividade contra bactérias Gram-negativas (Herald et al., 1983; Puupponen-Pimia et al., 2001).

Em um estudo in vitro preliminar, Carelli et al. (2011) determinou o potencial antimicrobiano da I. paraguariensis a partir do extrato da erva mate obtido por fluído supercrítico utilizando o dióxido de carbono (CO2) como solvente. Os microrganismos utilizados para a avaliação da atividade antimicrobiana foram a Acinetobacter baumannii, Escherichia coli, Pseudomonas aeruginosa, Staphylococcus aureus e Enterococcus faecalis, e o método escolhido foi a difusão em ágar. O extrato, por sua vez, demonstrou atividade contra duas bactérias apenas, a Staphylococcus aureus e Pseudomonas aeruginosa, inibindo o crescimento delas, além de demonstrar que a resposta inibitória pode ser dose dependente, uma vez que a suscetibilidade variou de acordo com a dosagem de extrato administrada. Sabe-se que Deans et al. (1987) \& Dorman et al. (2000), propõem que a ação inibitória da I. paraguariensis é mais ou menos eficiente frente a bactéria possivelmente em razão às singularidades da composição dos extratos que agem na composição celular dos microrganismos. Dessa forma, a importância desse estudo preliminar está na possibilidade de utilizar a I. paraguariensis, a partir de seus extratos, como um antibiótico.

Burris et al. (2012), ao avaliar extratos aquosos de erva-mate dialisados e liofilizados, constatou que eles são eficazes na inibição e na inativação de duas cepas de E. coli em um meio microbiológico e em suco de maçã (com pH ajustado para 6,0). Vários compostos que poderiam demonstrar atividade contra E. coli foram isolados - dentre eles ácido cafeico, ácido clorogênico, kaempferol, quercetina e rutina. Os dois primeiros, quando em sua forma pura, apresentaram atividade contra bactérias Gram-negativas; todavia, os três últimos não inibiram microrganismos. Extratos destilados de n-Hexano - linalool, aionona, b-ionona, a-terpineol, ácido octanóico, geraniol, 1-octanol, nerolidol, geranilactetona e eugenol - agiram como ótimos antimicrobianos, apesar de terem sido fracamente ativos contra E. coli.

O estudo de Martin, et al. (2013) avaliou a atividade antimicrobiana de extratos metanólicos e etanólicos da $I$. Paraguariensis contra patógenos alimentares como Staphylococcus aureus, Listeria monocytogenes, Salmonella enteritidis e Escherichia coli. A eficácia do extrato da erva mostrou melhor atividade em pH 6, 7 e 8; todos os microrganismos avaliados foram inibidos, exceto o E. coli. Ainda, foi possível perceber que o extrato etanólico apresentou maior inibição para $S$. aureus e S. enteriditis; para L. monocytogenes, os valores foram semelhantes.

Gonzalez-Gil et al. (2013) analisou, in vitro, a atividade biocida de extratos da erva mate liofilizados em Salmonella enteritidis e bactérias lácteas; nesse estudo, a erva-mate apresentou atividade biocida contra a Salmonella enteritidis, a qual foi muito sensível aos extratos da planta; entretanto, as bactérias probióticas tiveram um crescimento. Após esse resultado, um 
estudo in vivo foi conduzido o qual avaliou a erva-mate como um tratamento de aditivo alimentar, com o intuito de diminuir ou até inibir a transmissão horizontal e a colonização da Salmonella enteritidis. Nesse estudo, a erva-mate não foi capaz de reduzir a colonização de Salmonella enteritidis, porém, essa ineficácia pode ser explicada pela forma como o chá foi utilizado (cru), uma vez que nos estudos in vitro a avalição foi eficaz porque foram usados extratos concentrados de erva-mate.

Outra atividade antimicrobiana da erva-mate, conforme Tsai etal. (2015) encontrada é contra a Propionibacterium acne. Essa bactéria é do tipo Gram-positiva, se alimenta das secreções produzidas pelas glândulas sebáceas, e ao entrar em contato com os poros cutâneos, gera uma inflamação dos folículos pilosos; como consequência, origina lesões na pele, conhecidas como acnes. A Ilex paraguariensis, segundo os testes, foi capaz de inibir o crescimento da P. acne e esse resultado é positivo, já que mostra seu potencial para ser usada como agente terapêutico da acne. Além dos efeitos antimicrobianos da erva-mate, também foram registrados efeitos anti-inflamatórios.

Com os achados, Rempe, et al. (2015) em seu estudo determinou que extratos de erva-mate fracionados por proporções variáveis de metanol ou acetonitrila na água tiveram atividade variável contra Staphylococcus aureus e Staphylococcus aureus resistentes à meticilina e que o ácido 5-hidroxi-pipecólico, a quercetina, o ácido quínico, e um composto não identificado podem ser possíveis contribuintes para a atividade microbiana contra esse microrganismo.

Um polissacarídeo purificado da erva, estudado por Kungel, et al. (2018), teve efeitos antimicrobianos contra bactérias Gram-negativas (Enterobacter cloacae, Salmonella enteritidis e Salmonella typhimurium), bactérias Gram-positivas (Bacillus cereus, Micrococcus flavus, Staphylococcus aureus e Listeria monocytogenes) e contra Aspergillus fumigatus, Aspergillus versicolor, Aspergillus ochraceus, Aspergillus niger, Candida crusei, Penicillium funiculosum e Penicillium verrucosum var. ciclopium. O mesmo pôde ser considerado um forte inibidor contra B. cereus, M. flavus, E cloacae, $S$. enteritidis e $S$. typhimurium; entretanto, um inibidor fraco de E. coli. Falando dos bioensaios antifúngicos, o polissacarídeo pareceu ser um forte inibidor contra todos os fungos testados, exceto para A. niger.

Um estudo realizado por Noureddine et al. (2018) avaliou a atividade antibacteriana do extrato aquoso de caules e folhas da I. paraguariensis, extraídos a $70^{\circ} \mathrm{C}$, contra bactérias Gram-positivas e Gram-negativas, sendo elas: Acinetobacter baumannii, Pseudomonas aeruginosa, Staphylococcus aureus, Escherichia coli, Klebsiella pneumoniae, Enterococcus faecalis, Enterobacter agglomerans, Enterobacter aerogenes, Serratia marcesens, Escherichia coli e Proteus mirabilis. Nesse estudo, foi observada atividade bactericida contra todos os microrganismos testados, entretanto, ela variava entre as bactérias Gram-negativas e Gram-positivas, sendo essa atividade maior em bactérias Gram-positivas. De acordo com Bastos et al. (2007), essa variação de atividade antibacteriana pode ter relação com os componentes que são extraídos a cada temperatura de extração, uma vez que a $97^{\circ} \mathrm{C}$ o conteúdo fenólico é obtido em maior concentração, comparada a extração feita a $76^{\circ} \mathrm{C}$. Sendo assim, esse estudo é relevante pois mostra que a erva-mate pode ser utilizada no desenvolvimento de novos medicamentos antibacterianos.

\section{Conclusão}

Segundo os estudos avaliados, a planta Ilex paraguariensis possui ações antimicrobianas promissoras principalmente devido às suas extensas e ricas composições fotoquímicas. Os extratos da planta atuou em várias bactérias. O extrato etanólico demonstrou eficiente contra a Salmonella enteritidis in vitro (Girolometto et al., 2009; Martin, et al., 2013), assim como o metanólico (Martin, et al., 2013) e sem efeito no extrato de erva mate (Gonzalez-Gil et al., 2013), devendo ser alvo de pesquisas mais aprofundadas sobre o tema. Em relação a bactéria Escherichia coli, o extrato hidro alcoólico não apresentou atuação in vitro (De Biasi B. et al., 2009), ao contrário de extratos como o extrato aquoso (Burris et al. 2011; Burris et al.,2012; Noureddine et al., 2018), metanólico, etanólico (Martin, et al., 2013), compostos como ácido cafeico, cafeína ácido quínico rutina e teobromina (Puupponen-Pimia et al., 2001; Heck et al., 2007; Marques et al., 2009; Burris et al.2012). 
No que se refere ao S.aureus, houve atividade do extrato hidro alcoólico (De Biasi et al., 2009), etanólico (Girolometto et al., 2009; Martin, et al., 2013), metanólico (Martin, et al., 2013), aquoso, derivados de cafeoyl, ácido caffeoylshikimic, ácido feruloylquiniico, kaempferol, quercetina, ácido quínico (Rempe, et al.,2015), rutina e teobromina (Heck et al., 2007; Marques et al., 2009), ácidos cafeico e clorogênico (Herald et al., 1983; Puupponen-Pimia et al., 2001), extrato da erva mate (Carelli et al., 2011) e polissacarídeo purificado da planta (Kungel, et al., 2018).

Em relação a bactéria S.mutans o extrato de folhas verdes foi efetivo na enzima glicosiltransferase, sendo próspera para realizar mais estudos (Battagim, et al. (2011) a fim de visualizar o efeito antibiofilme, visto que nenhum estudo abordou especificamente o tema. A erva-mate apresentou atuação na bactéria Pseudomonas aeruginosa, sendo esta sensível ao extrato hidro alcoólico (De Biasi et al., 2009) e ao extrato da erva mate (Carelli et al., 2011). Além disso, a erva-mate demonstrou um efeito sob Propionibacterium acne, necessitando mais estudos para possível atuação contra a acne, doença que afeta jovens e influencia na sua qualidade de vida (Tsai et al.,2015. Vilar et al., 2015). Noureddine et al. (2018) mostrou que a planta possui atividade bactericida Gram- positivos e negativos, sendo maior em bactérias Gram-positivas, devido a maior conteúdo fenólico (Bastos et al.,2007).

Os artigos pesquisados não relatam a toxicidade da planta, sendo então necessárias pesquisas futuras sobre o tema bem como de efeitos adversos causados no organismo humano pelo consumo da planta, para que haja a aplicabilidade clínica com segurança das mesmas e de seu aproveitamento integral. Além disso, a atuação da Ilex paraguariensis no biofilme humano in situ também não foi estudada sendo uma questão de pesquisa a ser sanada em estudos futuros.

\section{Referências}

Battagim, J., De Souza, V. T., Sato Miyasaka, N. R., Da Silva Cunha, I. B., Sawaya, A. C., De Piloto Fernandes, A. M. A., Eberlin, M. N., Ribeiro, M. L., \& De Oliveira Carvalho, P. (2012). Comparative study of the effect of green and roasted water extracts of mate (Ilex paraguariensis) on glucosyltransferase activity of Streptococcus mutans. Journal of Enzyme Inhibition and Medicinal Chemistry, 27(2), 232-240. https://doi.org/10.3109/14756366.2011.585986

Baptista, D. Q., Santos, A. F. M., Aquino, M. H. C., Abreu, D. L. C., Rodrigues, D. P. \& Nascimento, E. R. (2018). Prevalência e susceptibilidade antimicrobiana de sorotipos de Salmonella spp. isolados de frangos vivos e carcaças no estado do Rio de Janeiro. Pesquisa Veterinária Brasileira, 38(7), 127885

Bezerra, M. S., Binotto, F. S., Richard, N. S. P. S., Bezerra, A. S., Marquezan, F. K. \& Marquezan, P. K. (2021). Avaliação medicinal e nutricional de três espécies de Plantas Alimentícias Não convencionais (PANCs): Uma revisão de literatura. Research, Society and Development. 10(5): e10410513401

Bezerra, M. S., Bezerra, A. S., Mendes, T. A. D. ., Brum, N. F., Souza, G. S. de . \& Marquezan, P. K. (2020). Atividade antimicrobiana e antibiofilme da Plantago major: uma revisão de literatura. Research, Society and Development, [S. 1.]. 9(9): e496997495. Doi: 10.33448/rsd-v9i9.7495

Burris, K. P., Davidson, P. M., Stewart, C. N., \& Harte, F. M. (2011). Antimicrobial Activity of Yerba Mate (Ilex paraguariensis) Aqueous Extracts against Escherichia coli O157:H7 and Staphylococcus aureus. Journal of Food Science, 76(6), 456-462. https://doi.org/10.1111/j.1750-3841.2011.02255.x

Burris, K. P., Davidson, P. M., Neal Stewart, C., Zivanovic, S., \& Harte, F. M. (2012). Aqueous extracts of yerba mate (Ilex paraguariensis) as a natural antimicrobial against Escherichia coli $\mathrm{O} 157: \mathrm{H} 7$ in a microbiological medium and $\mathrm{pH} 6.0$ apple juice. Journal of Food Protection, 75(4), 753-757. https://doi.org/10.4315/0362-028X.JFP-11-214

Brasil 2016. Surtos de doenças transmitidas por alimentos no Brasil. Ministério da Saúde.

Brum, N.F., Bezerra, M.S., Bezerra A. S., Souza, G. S. de. \& Marquezan, P. K. (2020). Atividade antibacteriana e antifúngica da herbácia Zingiber Officinale em odontologia: uma revisão de literatura. Research, Society and Development, 9(10): e6689109141. 10.33448/rsd-v9i10.9141.

Carelli, G., Macedo, S. M. D., Valduga, A. L., Corazza, M. L., Oliveira, J. V., Franceschi, E., VidaL, R., \& Jaskulski, M. R. (2011). Avaliação preliminar da atividade antimicrobiana do extrato de erva-mate (Ilex paraguariensis A. St.-Hil.) obtido por extração com CO2 supercrítico. Revista Brasileira de Plantas Medicinais, 13(1), 110-115. https://doi.org/10.1590/S1516-05722011000100016

De Biasi, B., Grazziotin, N. A., \& Hofmann Jr, A. E. (2009). Atividade antimicrobiana dos extratos de folhas e ramos da Ilex paraguariensis A. St.-Hil., Aquifoliaceae. Revista Brasileira de Farmacognosia, 19(2b), 582-585. https://doi.org/10.1590/s0102-695x2009000400013

Fejerskov, K (2011). Carie Dentária. A doença e seu tratamento clínico. (2a ed.), Elsevier

Francisco, K. S. F. (2010). Fitoterapia: Uma opção para o tratamento odontológico. Revista Saúde. 4(1): 18-24.

Girolometto, G., Avancini, C. A. M., Carvalho, H. H. C., \& Wiest, J. M. (2009). Antibacterial activity of yerba mate (Ilex paraguariensis A.St.-Hil.) extracts. 
Research, Society and Development, v. 10, n. 6, e41610615988, 2021

(CC BY 4.0) | ISSN 2525-3409 | DOI: http://dx.doi.org/10.33448/rsd-v10i6.15988

Revista Brasileira de Plantas Medicinais, 11(1), 49-55. https://doi.org/10.1590/s1516-05722009000100009

Gonzalez-Gil, F., Diaz-Sanchez, S., Pendleton, S., Andino, A., Zhang, N., Yard, C., Crilly, N., Harte, F., \& Hanning, I. (2014). Yerba mate enhances probiotic bacteria growth in vitro but as a feed additive does not reduce Salmonella Enteritidis colonization in vivo. Poultry Science, 93(2), 434-440. https://doi.org/10.3382/ps.2013-03339

Kungel, P. T. A. N., Correa, V. G., Corrêa, R. C. G., Peralta, R. A., Soković, M., Calhelha, R. C., Bracht, A., Ferreira, I. C. F. R., \& Peralta, R. M. (2018). Antioxidant and antimicrobial activities of a purified polysaccharide from yerba mate (Ilex paraguariensis). International Journal of Biological Macromolecules, 114(2017), 1161-1167. https://doi.org/10.1016/j.ijbiomac.2018.04.020

Noureddine, T., El Husseini, Z., Nehme, A., \& Abdel-Massih, R. M. (2018). Antibacterial activity of Ilex paraguariensis (Yerba mate) against gram-positive and gram-negative bacteria. Journal of Infection in Developing Countries, 12(9), 712-719. https://doi.org/10.3855/jidc.10380

Pereira A. S. (2018). Metodologia da pesquisa científica. UFSM

Prado Martin, J. G., Porto, E., de Alencar, S. M., da Glória, E. M., Corrêa, C. B., \& Ribeiro Cabral, I. S. (2013). Actividad antimicrobiana de la yerba mate (Ilex paraguariensis St. Hil.) contra patógenos alimentarios. Revista Argentina de Microbiologia, 45(2), 93-98. https://doi.org/10.1016/S0325-7541(13)700063

Rempe, C. S., Burris, K. P., Woo, H. L., Goodrich, B., Gosnell, D. K., Tschaplinski, T. J., \& Stewart, C. N. (2015). Computational ranking of yerba mate small molecules based on their predicted contribution to antibacterial activity against methicillin-resistant staphylococcus aureus. PLoS ONE, 10(5), 1-18. https://doi.org/10.1371/journal.pone.0123925

Silva, M. V. (2012). Infecções do trato urinário por Escherichia coli uropatogênica: uma revisão. 46 f. Tese (Pós-Graduação Lato Sensu em Microbiologia aplicada às Ciências da Saúde) - Universidade Federal de Minas Gerais.

Tsai, T. H., Tsai, T. H., Wu, W. H., Tseng, J. T. P., \& Tsai, P. J. (2010). In vitro antimicrobial and anti-inflammatory effects of herbs against Propionibacterium acnes. Food Chemistry, 119(3), 964-968. https://doi.org/10.1016/j.foodchem.2009.07.062

Vilar, G. N., Santos, L. A. \& Sobral Filho, J. F. (2015). Qualidade de vida, autoestima e fatores psicossociais em adolescentes com acne vulgar. Anais Brasileiros de Dermatologia, 90 (5), 622-9. 